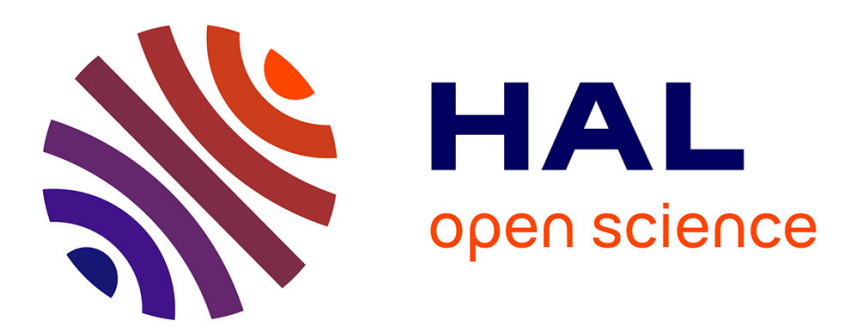

\title{
Effect of 3rd-degree gravity harmonics and Earth perturbations on lunar artificial satellite orbits
}

S. Tzirti, K. Tsiganis, H. Varvoglis

\section{To cite this version:}

S. Tzirti, K. Tsiganis, H. Varvoglis. Effect of 3rd-degree gravity harmonics and Earth perturbations on lunar artificial satellite orbits. Celestial Mechanics and Dynamical Astronomy, 2010, 108 (4), pp.389-404. 10.1007/s10569-010-9313-3 . hal-00584606

\section{HAL Id: hal-00584606 https://hal.science/hal-00584606}

Submitted on 9 Apr 2011

HAL is a multi-disciplinary open access archive for the deposit and dissemination of scientific research documents, whether they are published or not. The documents may come from teaching and research institutions in France or abroad, or from public or private research centers.
L'archive ouverte pluridisciplinaire HAL, est destinée au dépôt et à la diffusion de documents scientifiques de niveau recherche, publiés ou non, émanant des établissements d'enseignement et de recherche français ou étrangers, des laboratoires publics ou privés. 


\title{
Effect of $3^{\text {rd }}$-degree gravity harmonics and Earth perturbations on lunar artificial satellite orbits
}

\author{
S. Tzirti · K. Tsiganis - H. Varvoglis
}

Received: date / Accepted: date

\begin{abstract}
In a previous work we studied the effects of (I) the $J_{2}$ and $C_{22}$ terms of the lunar potential and (II) the rotation of the primary on the critical inclination orbits of artificial satellites. Here, we show that, when $3^{r d}$-degree gravity harmonics are taken into account, the long-term orbital behavior and stability are strongly affected, especially for a non-rotating central body, where chaotic or collision orbits dominate the phase space. In the rotating case these phenomena are strongly weakened and the motion is mostly regular. When the averaged effect of the Earth's perturbation is added, chaotic regions appear again for some inclination ranges. These are more important for higher values of semi-major axes. We compute the main families of periodic orbits (POs), which are shown to emanate from the 'frozen eccentricity' and 'critical inclination' solutions of the axisymmetric problem (' $J_{2}+J_{3}$ '). Although the geometrical properties of the orbits are not preserved, we find that the variations in $e, I$ and $g$ can be quite small, so that they can be of practical importance to mission planning.
\end{abstract}

Keywords Lunar artificial satellites $\cdot 3^{r d}$-degree gravity coefficients $\cdot 3^{\text {rd }}$-body effect

\section{Introduction}

During the past decades there has been extensive study of satellite orbits around an oblate primary. The consideration of the $J_{2}$ term of the spherical harmonic expansion of the potential leads to a critical value of the inclination, equal to $63^{\circ} .43$, for which the orbit does not precess secularly (Garfinkel 1973; Hughes 1981; Coffey, Deprit and Miller 1986; Jupp 1988). For the case of the Earth, Cutting et al. (1978) examined the conditions under which the long-periodic argument of pericentre and eccentricity remain both constant with the additional effect of the $J_{3}$ term (frozen orbits). Coffey et al. (1994) searched for frozen orbits close to an Earth-like planet, including up to

Section of Astrophysics Astronomy \& Mechanics, Department of Physics,

University of Thessaloniki, 54124 Thessaloniki, Greece

Tel.: +30-2310-998135

E-mail: stzir@physics.auth.gr 
$6^{\text {th }}$-degree zonal coefficients, while Knežević and Milani (1998) took into consideration all the available zonal coefficients for the case of a polar lunar orbiter. The tesseral and sectorial coefficients were, in all cases, neglected, as their values were considered too small to affect the long term orbital behavior or the critical inclination value.

The situation for the Moon is different than for the Earth, as the $C_{22}$ coefficient is only nine times smaller than $J_{2}$. This problem was first examined by De Saedeleer and Henrard (2006), who showed that the value of the critical inclination depends on the initial phase of the ascending node. In their derivation the rotation of the Moon and the time variation of the averaged inclination were neglected, most likely both considered to be insignificant on the assumed time scale. In a previous work (Tzirti et al. 2009) we showed that, under the combined effect of $J_{2}$ and $C_{22}$ harmonics, the averaged inclination does not remain constant but performs long periodic oscillations. The time evolution of the inclination causes variations in the argument of pericentre, of similar amplitude. Under those circumstances, the term 'critical inclination' becomes meaningless. Instead, we used the term 'quasi-critical' for the solutions in which the argument of pericentre librates. On the other hand we showed that the strong dependence of the 'critical inclination' on the longitude of the ascending node, found by De Saedeleer and Henrard (2006), is considerably weakened when the rotation of the Moon is taken into account.

Besides $C_{22}$, even the higher-degree harmonics are important for the case of Moon. In this work we take into account perturbations up to $3^{r d}$-degree. So, we include the ' $J_{3}$ ' effect and the non-axisymmetric part as well. The averaged system has now two degrees of freedom and the long-term stability of the orbits is modified. As we show below, for a non-rotating Moon, extended regions of chaotic motion or regions of collision orbits (due to the large amplitude oscillations of the eccentricity) occupy a large portion of phase space. However, the rotation of the Moon smooths out these phenomena. The motion appears to be mostly regular for all semi-major axes and orientations studied. We compute the main families of stable periodic orbits, which are shown to emanate from the POs of the ' $J_{2}+J_{3}$ ' axisymmetric case. There are two families, one corresponding to the low- $e$ 'frozen' orbits $(\forall I)$ and the other to the large- $e$ 'critical inclination' solution (at $I \approx 63^{\circ} .5$ ).

For distances greater than $3 R$ (where $R$ is the mean equatorial radius of the Moon), it is necessary to consider the effect of the Earth (as e.g. in De Saedeleer 2006b), which changes drastically the orbital behavior, especially at high inclinations. We find that in some regions of phase space the motion seems to be chaotic or to lead to collision with the surface of the Moon. For relatively large semi-major axis values, the effect of the Earth becomes dominant, while for orbits close to the Moon, the $3^{r d}$-degree gravitational harmonics seem to play the decisive role. In both cases we examine the distribution of the main families of POs (in the averaged problem).

\section{Hamiltonian of the problem and equations of motion}

\subsection{Gravity harmonics of the Moon}

We consider an artificial satellite in orbit around the Moon. We use a rotating frame whose origin is at the center of the Moon, the $x$ axis passes through the longest lunar 


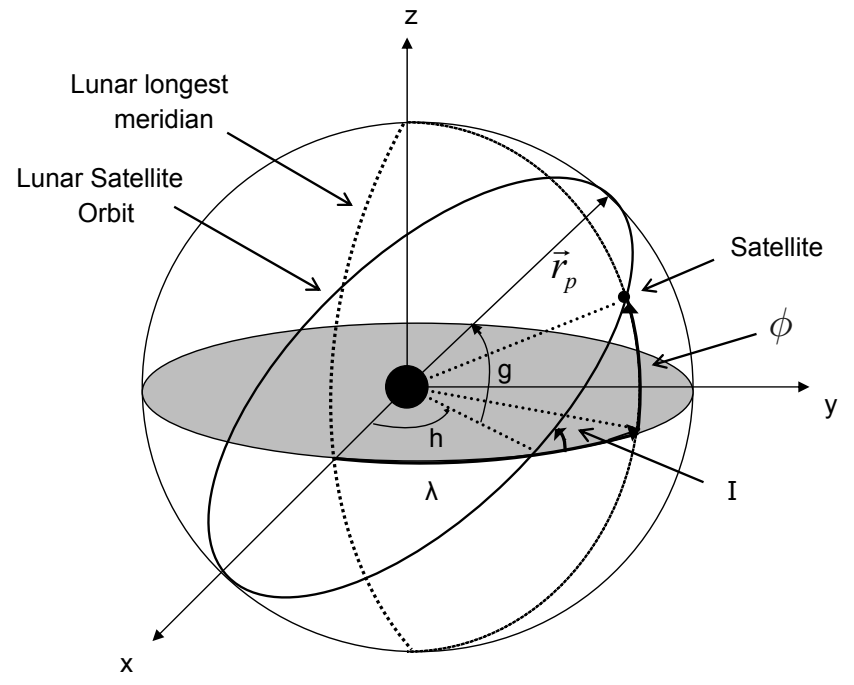

Fig. 1 Orientation of the orbit in space

meridian and the $x-y$ plane coincides with the lunar equatorial plane. This frame rotates at the rate of the Moon's mean synchronous rotation, $n_{M}$ (see Fig. 1). The potential, written in selenographic coordinates, has the general form (see e.g. Vallado 2001; Sidi 2002; Bertotti et al. 2003)

$$
V=-\frac{\mu}{r} \sum_{n=0}^{\infty}\left(\frac{R}{r}\right)^{n} \sum_{m=0}^{n} P_{n m}(\sin \phi)\left[C_{n m} \cos m \lambda+S_{n m} \sin m \lambda\right]
$$

where $\mu=\mathcal{G M}, R$ is the mean equatorial radius of the Moon, $(\lambda, \phi)$ are the selenographic longitude and latitude, respectively, $r$ the selenocentric distance of the satellite, $C_{n m}$ and $S_{n m}$ the non-normalized gravity coefficients and $P_{n m}$ the Legendre polynomials of degree $n$ and order $m$. For $m=0$ we get the zonal harmonic coefficients, for which we will use the notation $J_{n}=-C_{n 0}$. The numerical values used for the constants are $\mathcal{G}$ $=6.6739 \times 10^{-11} \mathrm{~m}^{3} \mathrm{~kg}^{-1} \mathrm{~s}^{-2}, M=7.349 \times 10^{22} \mathrm{~kg}$ and $R=1737530 \mathrm{~m}$. To simplify the calculations, we use dimensionless units, by taking $\mu=1$ and $a_{r e s_{1-1}}=1$, where $a_{r e s_{1-1}}(=88459747.12676102 \mathrm{~km})$ represents the semi-major axis value for which the period $T$ of the satellite is in $1-1$ resonance with the rotational period of the Moon in the unperturbed problem. In this system of units $T=2 \pi$. The numerical results presented in this paper follow the above unit conventions.

In this paper we consider the effect of terms up to $3^{r d}$-degree, omitting $C_{21}, S_{21}$ and $S_{22}$, whose values are at least three orders of magnitude smaller than $C_{22}$ and have considerable error bars (see Table 1 or De Saedeleer 2006a - Tab. 1.5. Note, however, that the values come from different sources: Bills 1980 for $2^{n d}$-degree and Konopliv 1998 for $3^{r d}$-degree). The inclusion of these terms would complicate the equations, while it would give negligible improvement to the results. It is already obvious that the 
Table 1 Gravitational parameters values and uncertainties (De Saedeleer 2006a)

\begin{tabular}{ll}
\hline parameter & (value \pm uncertainty) $\times 10^{-6}$ \\
\hline$J_{2}$ & $202.43 \pm 1.14$ \\
$C_{21}$ & $-0.0035 \pm 0.0058$ \\
$C_{22}$ & $22.26 \pm 0.13$ \\
$S_{21}$ & $-0.00098 \pm 0.0045$ \\
$S_{22}$ & $0.0108 \pm 0.0020$ \\
$J_{3}$ & $8.476 \pm 0.017$ \\
$C_{31}$ & $28.437 \pm 0.0081$ \\
$C_{32}$ & $4.846 \pm 0.002$ \\
$C_{33}$ & $1.7132 \pm 0.0006$ \\
$S_{31}$ & $5.9022 \pm 0.0048$ \\
$S_{32}$ & $1.6709 \pm 0.0021$ \\
$S_{33}$ & $-0.249 \pm 0.0006$ \\
\hline
\end{tabular}

leading $3^{r d}$-degree terms are $J_{3}$ and $C_{31}$. In the following, we will use the parameter values given in Table 1.

The Hamiltonian of the problem has the following form:

$$
\mathcal{H}=\mathcal{H}_{0}+\mathcal{H}_{J_{2}}+\mathcal{H}_{C_{22}}+\mathcal{H}_{J_{3}}+\sum_{j=1}^{3} \mathcal{H}_{C_{3 j}}+\sum_{j=1}^{3} \mathcal{H}_{S_{3 j}}+\mathcal{H}_{n_{M}}
$$

where $\mathcal{H}_{0}=u^{2} / 2-\mu / r$ is the Keplerian part, $\mathcal{H}_{\mathcal{J}_{3}}, \mathcal{H}_{\mathcal{C}_{3 j}}$ and $\mathcal{H}_{\mathcal{S}_{3 j}}$ correspond to the $3^{r d}$-degree gravity harmonics and $\mathcal{H}_{n_{M}}=-n_{M} p_{\lambda}\left(p_{\lambda}\right.$ is the momentum conjugate to $\lambda$ ) describes the rotation of the Moon. In selenographic coordinates, $\mathcal{H}$ will contain powers of the distance $r$, powers of $\sin \phi$, coming from the Legendre polynomials $P_{n m}$, and $\cos m \lambda, \sin m \lambda$, which are also easily expressed as powers of $\cos \lambda$ and $\sin \lambda$. The angles $\phi, \lambda$ and the distance $r$ can be written as functions of the semi-major axis, $a$, the eccentricity, $e$, the inclination, $I$, of the orbital plane relative to the equatorial one, the longitude of the ascending node in the rotating system, i.e. $h=\Omega-n_{M} t$, the argument of pericentre, $g$, and the true anomaly, $f$, using the relations (7.10) from De Saedeleer (2006a):

$$
\begin{gathered}
\sin \phi=s Y \\
X=\cos \phi \cos (\lambda-h) \\
\cos \phi=c Y \sin (\lambda-h)+X \cos (\lambda-h)
\end{gathered}
$$

where $s=\sin I, c=\cos I, Y=\sin (f+g), X=\cos (f+g)$.

After performing the trigonometric expansions in (4) and (5), we can solve the first of them for $\sin \lambda$ and replace it in the second one, which can now be solved for $\cos \lambda$. Using the relations $X^{2}=1-Y^{2}, s^{2}=1-c^{2}$ and $\cos \phi=\sqrt{1-s^{2} Y^{2}}$ where it is essential, we can derive the simplified expressions for $\cos \lambda$ and $\sin \lambda$ as functions of the orbital elements 
Table $23^{r d}$ degree terms that appear in the Hamiltonian. $K=3 \mu e R^{3} /\left[16 a^{4}\left(1-e^{2}\right)^{5 / 2}\right]$, $c=\cos I, s=\sin I$

\begin{tabular}{lll}
\hline parameter & $K_{3 j}^{ \pm}$ & $K_{3 j}^{\prime \pm}$ \\
\hline$C_{31}$ & $K\left(1 \pm 11 c-5 c^{2} \mp 15 c^{3}\right)$ & 0 \\
$C_{32}$ & 0 & $10 K\left(-s \pm 2 c s+3 c^{2} s\right)$ \\
$C_{33}$ & $30 K\left(1 \pm c-c^{2} \mp c^{3}\right)$ & 0 \\
$S_{31}$ & 0 & $\pm K\left(1 \pm 11 c-5 c^{2} \mp 15 c^{3}\right)$ \\
$S_{32}$ & $\mp 10 K\left(-s \pm 2 c s+3 c^{2} s\right)$ & 0 \\
$S_{33}$ & 0 & $\pm 30 K\left(1 \pm c-c^{2} \mp c^{3}\right)$ \\
\hline
\end{tabular}

$$
\begin{aligned}
& \cos \lambda=\frac{X \cos h-c Y \sin h}{\sqrt{1-s^{2} Y^{2}}} \\
& \sin \lambda=\frac{X \sin h+c Y \cos h}{\sqrt{1-s^{2} Y^{2}}}
\end{aligned}
$$

Substituting $\cos \lambda$ and $\sin \lambda$ in $\mathcal{H}$, the latter becomes a function of $[a, e, I, f, h, g]$. Following Roy (1982) we can derive the averaged Hamiltonian to first order in $\left(J_{n}\right.$, $\left.C_{n m}, S_{n m}\right)$, by integrating $\mathcal{H}$ over the mean anomaly, using standard trigonometric formulas and applying the following change of variables:

$$
d M=\frac{r^{2}}{a^{2}\left(1-e^{2}\right)^{1 / 2}} d f
$$

The steps described above can be applied to obtain the averaged Hamiltonian for up to every term of degree $n$ and order $m$ in the perturbing potential. In our case, the averaged terms of (2) are given below:

$$
\begin{gathered}
\overline{\mathcal{H}}_{0}=-\frac{\mu}{2 a}, \quad \overline{\mathcal{H}}_{n_{M}}=-n_{M} \sqrt{\mu a\left(1-e^{2}\right)} c \\
\overline{\mathcal{H}}_{J_{2}}=\frac{\mu J_{2} R^{2}\left(1-3 c^{2}\right)}{4 a^{3}\left(1-e^{2}\right)^{3 / 2}}, \quad \overline{\mathcal{H}}_{C_{22}}=-\frac{3 \mu C_{22} R^{2} s^{2}}{2 a^{3}\left(1-e^{2}\right)^{3 / 2}} \cos 2 h \\
\overline{\mathcal{H}}_{J_{3}}=2 J_{3} K\left(-4+5 s^{2}\right) s \sin g \\
\overline{\mathcal{H}}_{C_{3 j}}=C_{3 j}\left[K_{3 j}^{+} \cos (g+j h)+K_{3 j}^{-} \cos (g-j h)+\right. \\
\left.K_{3 j}^{\prime+} \sin (g+j h)+K_{3 j}^{\prime-} \sin (g-j h)\right] \\
\overline{\mathcal{H}}_{S_{3 j}}=S_{3 j}\left[K_{3 j}^{+} \cos (g+j h)+K_{3 j}^{-} \cos (g-j h)+\right. \\
\left.K_{3 j}^{\prime+} \sin (g+j h)+K_{3 j}^{\prime-} \sin (g-j h)\right]
\end{gathered}
$$

where $K_{3 j}^{ \pm}$and $K_{3 j}^{\prime \pm}$ are given in Table 2 . 
Before proceeding to the inclusion of a $3^{r d}$-body perturbation and analyzing the methods of work, it would be of interest to have a look at the solutions of the axisymmetric problem (the averaged Hamiltonian contains only the terms $J_{2}$ and $J_{3}$ ). In that way, we will be able to examine how the asymmetry introduced by the additional sectorial and tesseral terms affects the phase space and POs. Following Vallado (2001) $\dot{g}$ and $\dot{e}$ will be:

$$
\dot{g}=-\frac{3 n_{M} J_{2}\left(1-5 c^{2}\right)}{4\left(1-e^{2}\right)^{2}}\left(\frac{R}{a}\right)^{2} N(e, I, g),
$$

where

$$
N(e, I, g)=1+\frac{J_{3}}{2 J_{2}}\left(\frac{R}{a}\right)\left(\frac{1}{1-e^{2}}\right)\left(\frac{s^{2}-e^{2} c^{2}}{s}\right) \frac{\sin g}{e}
$$

and

$$
\dot{e}=\frac{3 J_{3}}{8} \frac{n_{M}}{\left(1-e^{2}\right)^{2}}\left(\frac{R}{a}\right)^{3} s\left(1-5 c^{2}\right) \cos g
$$

One can reach the same results using Eqs. (21) in the next page. A periodic solution $(\dot{g}=0, \dot{e}=0)$ is obtained in two cases:

(a) for $I=63^{\circ} .43=$ const. ('critical inclination' orbits), so that Eqs. (14) and (16) become both equal to zero. This solution occurs for every value of $e_{0}$ and $g_{0}$ and it already exists in the $J_{2}$ problem

(b) for $g= \pm \pi / 2$, which satisfies $\dot{e}=0$ and appropriate values of $e_{0}, I_{0}$, so that $N(e, I, \pm \pi / 2)=0$ or equally $\dot{g}=0$ ('frozen eccentricity' orbits). For $I=0$ or $e=0$ a singularity appears in Eq. (15), which does not allow us to give initial conditions exactly equal to these values.

Frozen satellite orbits have been also numerically computed by Lara et al. (1995), who included in their model zonal coefficients up to order 9, while Abad et al. (2009) developed an analytic model for locating frozen orbits keeping zonal harmonics $J_{2}$ and $J_{7}$. Other work in the same direction is that of Elipe and Lara (1997), in which, starting from results on frozen orbits in the zonal problem, they examine their continuation under the inclusion of the $C_{22}$ term. Moreover, Lara et al. (2009) computed frozen orbits in the zonal problem and they compared them to results from analytic solutions.

We now turn to the computation of the averaged perturbation exerted by the Earth.

2.2 The effect of the Earth as a $3^{r d}$-body perturbation

As we go further away from the surface of the Moon, the effect of the Earth, as a $3^{r d}$ body perturbation, cannot be neglected. To include the interaction with the Earth, we work in a rotating frame with the Moon at its origin (Fig. 2). The perturbing potential arising from Earth has the form (see e.g. Murray and Dermott 1999)

$$
V_{\text {Earth }}=-\frac{\mu^{\prime}}{\left|\mathbf{r}-\mathbf{r}^{\prime}\right|}+\mu^{\prime} \frac{\mathbf{r} \cdot \mathbf{r}^{\prime}}{\mathbf{r}^{\prime 3}}
$$




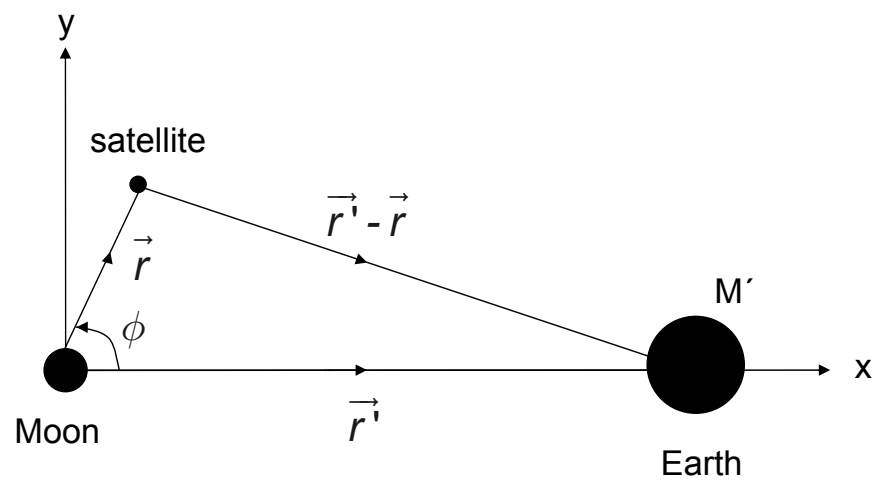

Fig. 2 The position vectors of the satellite and the Earth with respect to the Moon

where $\mu^{\prime}=G M_{\text {Earth }}\left(M_{\text {Earth }}=5.9736 \times 10^{24} \mathrm{~kg}\right)$ and $\mathbf{r}, \mathbf{r}^{\prime}$ are the position vectors of the satellite and the Earth relative to the Moon. $V_{\text {Earth }}$ can be expressed as a series of Legendre Polynomials $P_{k}$

$$
V_{\text {Earth }}=-\frac{\mu^{\prime}}{d} \sum_{k=2}^{\infty}\left(\frac{r}{d}\right)^{k} P_{k}(\cos \phi)
$$

from which we will only keep the $k=2$ term. We assume circular motion of the Earth around the Moon at the mean distance $d=\left\langle r^{\prime}\right\rangle=a_{0}\left(1+e_{0}^{2} / 2\right)$, where $a_{0}=384,400$ $\mathrm{km}$ and $e_{0}=0.0549$, and that the orbital plane is the Moon's equatorial plane. In this way the selenographic latitude, $\phi$, is written as a function of the angles $f, g, h$ by using the first term (in our case the other two terms are considered equal to zero because of the previous assumptions) of Eq. (8.10) from De Saedeleer (2006a).

$$
\cos \phi=X \cos h-c Y \sin h
$$

After expanding the trigonometric sums in $X(=\cos (f+g))$ and $Y(=\sin (f+g))$, using standard formulas for expressing $\cos f, \sin f$ and $r$ as functions of the eccentric anomaly $(E)$ and changing consistently the variable from $M$ to $E$, we obtain the first order averaged Hamiltonian by integrating over $E$. The choice of working with $E$ simplifies the calculations. The final result is

$$
\begin{aligned}
\mathcal{H}_{\text {Earth }}= & \frac{\mu^{\prime} a^{2}}{16 d^{3}}\left\{\left(1-3 c^{2}\right)\left(2+3 e^{2}\right)-3 s^{2}\left[5 e^{2} \cos (2 g)+\left(2+3 e^{2}\right) \cos (2 h)\right]\right. \\
& \left.-\frac{15 e^{2}}{2}\left[(-1+c)^{2} \cos [2(g-h)]-(1+c)^{2} \cos [2(g+h)]\right]\right\}
\end{aligned}
$$

and it formally agrees with that of De Saedeleer (2006b).

In order to find the equations of motion, either with or without the inclusion of Earth, we use the well-known equations

$$
\dot{g}=\partial \overline{\mathcal{H}} / \partial G, \quad \dot{G}=-\partial \overline{\mathcal{H}} / \partial g
$$




$$
\dot{h}=\partial \overline{\mathcal{H}} / \partial H, \quad \dot{H}=-\partial \overline{\mathcal{H}} / \partial h
$$

where $[L, G, H]$ are the Delaunay actions.

$$
L=\sqrt{\mu a}, \quad G=\sqrt{\mu a\left(1-e^{2}\right)}, \quad H=\sqrt{\mu a\left(1-e^{2}\right)} \cos I
$$

In the following, we focus our study on three models: the ' $3 \mathrm{G}$ ' model, which contains only up to $3^{\text {rd }}$-degree gravity harmonics, the ' $3 \mathrm{G}+\mathrm{R}$ ' model, which contains the additional effect of the rotation of the Moon and, finally, the more complete ' $3 \mathrm{G}+\mathrm{R}+\mathrm{E}$ ' model that also introduces the effect of the Earth as a $3^{\text {rd }}$ - body perturbation. In all models, the dynamical system has two degrees of freedom. To study the global behavior of orbits, we will use Poincaré maps, as well as Fast Lyapunov Indicator (FLI) maps (Froeschlé et al 1997). Finally, we will compute the main families of POs and analyze their geometrical and dynamical properties.

\section{Poincaré sections and FLI maps}

The Poincaré maps presented here are taken on the $G-g$ plane, defined from the section $h=\pi$ and $\dot{h}<0$. Note that $G$ is dimensionless in the system of units described above. The choice $h=\pi$ is just indicative. A different choice of $h$ for the section plane (i.e. $h=0$ ) would give similar results to the $h=\pi$. The section is defined for $\dot{h}<0$, because $h$ decreases as time increases. The choice of the opposite sign would give no points on the section. For given initial conditions $\left(a, g_{0}, G_{0}, h_{0}\right)$ and 'energy' values, we use a Newton-Raphson method to determine $H_{0}$. The equations of motion are solved using a $4^{t h}$-order Runge-Kutta scheme with step equal to $T / 10$, where $T$ is the period of the satellite in the unperturbed problem (the order of magnitude of $T$ is several hours for the semi-major axis values considered here. For example, $T=2.23$ hours for $a=2000 \mathrm{~km}$ and $T=6.3$ hours for $a=4000 \mathrm{~km}$ ). To achieve better accuracy on the crossing point of the section surface, we first find a point immediately after the crossing, we change the independent variable to $h$ (the equations of motion now are $\left.t^{\prime}(h)=d t / d h=1 / \dot{h}, g^{\prime}(h)=\dot{g} / \dot{h}, H^{\prime}(h)=\dot{H} / \dot{h}, G^{\prime}(h)=\dot{G} / \dot{h}\right)$ and integrate backwards until $h=\pi$ with accuracy $10^{-12}$. As the energy level and $h$ are constant on each section, each curve on the map corresponds to a different inclination range.

The computation of FLI allows us to discriminate ordered and chaotic motion or even resonant and non resonant regular orbits on a relatively short time interval. Here we use the revisited definition given in Lega and Froéschle (2001). FLI maps are not useful for integrable systems, since the motion is regular in the whole phase space. For given initial $a, g_{0}$ and $h_{0}$, we chose a $50 \times 90$ grid in $\left(e_{0}, I_{0}\right)$ in the range $0<e<e_{c}$ and $0<I<180^{\circ}$, where $e_{c}$ is the collision eccentricity. For each set of initial conditions, FLI is equal to the logarithm of the maximum value that the norm of the tangent vector reaches up to time $t$. As the evolution of the system is slowed when we increase the semi-major axis value, we selected to integrate for $t=1500$ lunar months. This time scale is quite large and it exceeds the typical duration of a lunar mission, but it is essential for semi-major axis values until $a=12 R$, for which the characteristics of the orbits (for example chaotic behavior) are exhibited after some hundreds of lunar months. Regular orbits close to resonance $(F L I \leq 5)$ are represented by black color on the FLI maps, while the white colored regions indicate chaotic or collision regions. Below we discuss the results for each model separately. 
' $3 G$ ' model: As it was shown in Tzirti et al. (2009), under the combined effect of $J_{2}$ and $C_{22}$ terms of the lunar potential the orbits remain regular. This is because the system is effectively reduced to one degree of freedom, since in this case $G$ is an integral of motion. The inclusion of the $3^{r d}$-degree terms, however, seems to change significantly the secular orbital behavior. Poincaré sections and FLI maps contain extended chaotic regions, encompassing regions where regular orbits have secular oscillation amplitude in $e$ large enough to lead to collision on the surface of the Moon (collision regions - see Figs. 3, 4a). The collision limits are indicated on the Poincaré sections by horizontal, solid lines. The semi-major axis values used in this model are $a=\left(2 \times 10^{3}, 3 \times 10^{3}\right.$, $\left.4 \times 10^{3}\right) \mathrm{km}$. For $a=2 \times 10^{3} \mathrm{~km}$, chaotic and collision regions dominate the phase space to such an extent, that regular regions are hardly detectable (that is why this is not shown). It should be noted that points in Fig. 3b, that seem to form an unusual series crossing the other lines, belong to different curves. The $3^{r d}$-degree terms introduce an asymmetry relative to the $I=90^{\circ}$ plane (Fig. 4a). This practically means that two orbits with initial inclinations $I$ and $180^{\circ}-I$ do not exhibit the same behavior (see Fig. 4a).

' $3 G+R$ ' model: In this case the motion seems to be mostly regular for all semi-major axes studied, as the effect of the lunar rotation smooths out the chaotic behavior. Terms depending on $h$ are now dominated by rotation, so the asymmetry with respect to $I=90^{\circ}$ is strongly weakened. Poincaré maps show the existence of stable POs at small eccentricities $(<0.01)$ for any value of inclination (Fig. 5). These correspond to the 'frozen- $e$ ' solution of the axisymmetric problem. For inclinations close to $63^{\circ} .5$ or $116^{\circ} .5$, another family of eccentric POs seems to bifurcate from the 'critical inclination' solution of the ' $J_{2}+J_{3}$ ' problem.

' $3 G+R+E$ ' model: We studied orbits with semi-major axes in the range $R+100 \mathrm{~km}$ $\leq a \leq 20000 \mathrm{~km}$ under the combined effects of $3^{r d}$-degree gravity harmonics and the perturbation of Earth. The characteristics of the motion are different close to and far from the surface of the Moon, where the lunar gravity harmonics or the $3^{\text {rd }}$-body effects are dominant, respectively. In Fig. 6 we present the Poincaré sections for $a=3000 \mathrm{~km}$ and $a=10000 \mathrm{~km}$, for three different inclination ranges (again it is chosen $h_{o}=\pi$ for the surfaces of section. This value is just indicative. The same analysis performed for other values of $h_{o}$ gives similar results). For $a=3000 \mathrm{~km}$ and $I<58^{\circ}$, the motion appears to be regular and there are two main islands where motion does not lead to collision, centered around $g_{0} \approx \pm \pi / 2$ (Fig. 6a). For $58^{\circ}<I<78^{\circ}$, the orbits are either chaotic or lead to collision, due to regular secular oscillations (Fig. 6c). The main islands do not anymore correspond to orbits with acceptable eccentricity values, so there are no POs corresponding to 'collision-free' motion (Fig. 4b). For inclinations close to $90^{\circ}$ (near-polar orbits), the phase space mainly consists of regular orbits and two main islands of 'collision-free' motion appear again, but now at $g_{0} \approx 0, \pi$ (Fig. $6 \mathrm{e}$ ). For $a>3000 \mathrm{~km}$ the structure of phase space is almost the same as that for $a<3000$ $\mathrm{km}$ only for $I<60^{\circ}$ (Fig. 6b). As the inclination increases, the main islands disappear and the phase space is dominated by chaotic regions that become more extensive as the inclination approaches $90^{\circ}$ (Figs. 6d, 6f). 


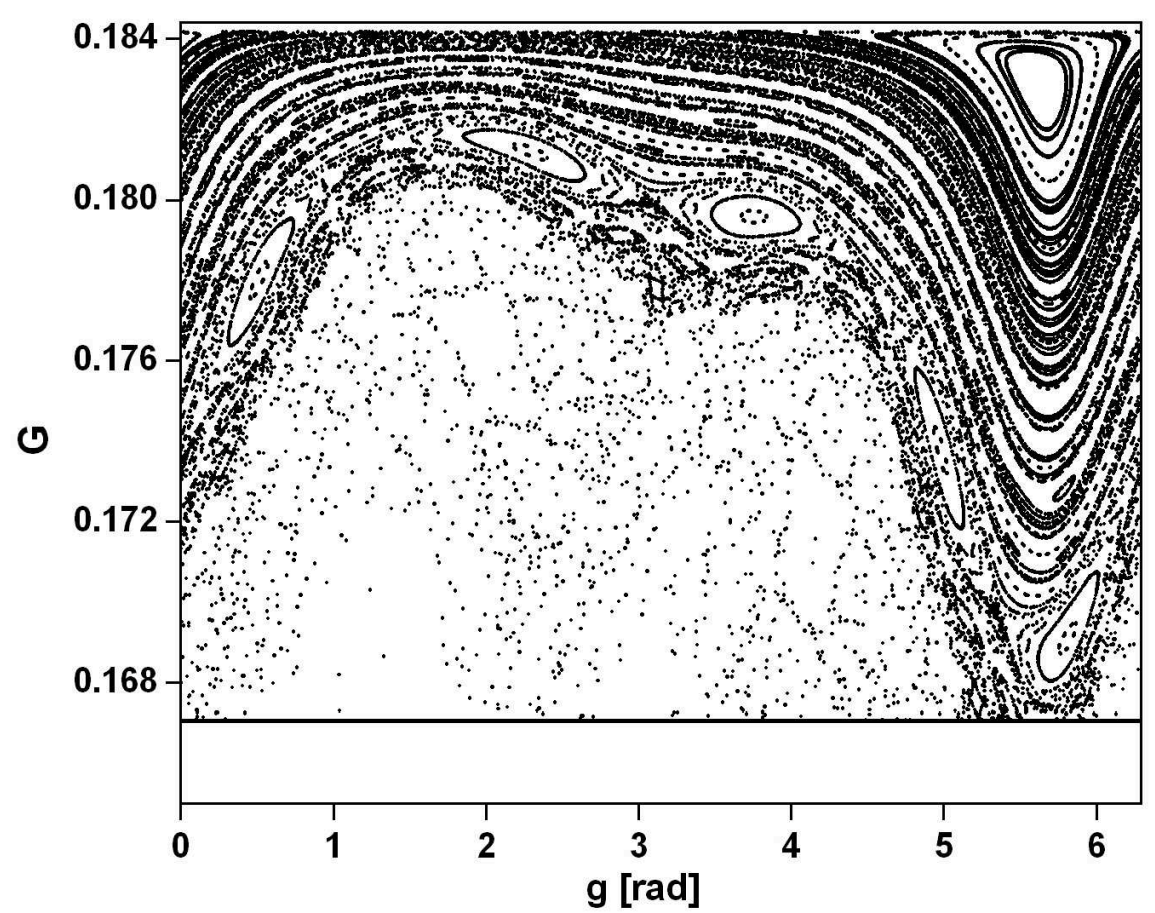

a. $\alpha=3000 \mathrm{~km}, 40^{\circ}<\mathrm{I}_{\mathrm{o}}<47^{\circ}$

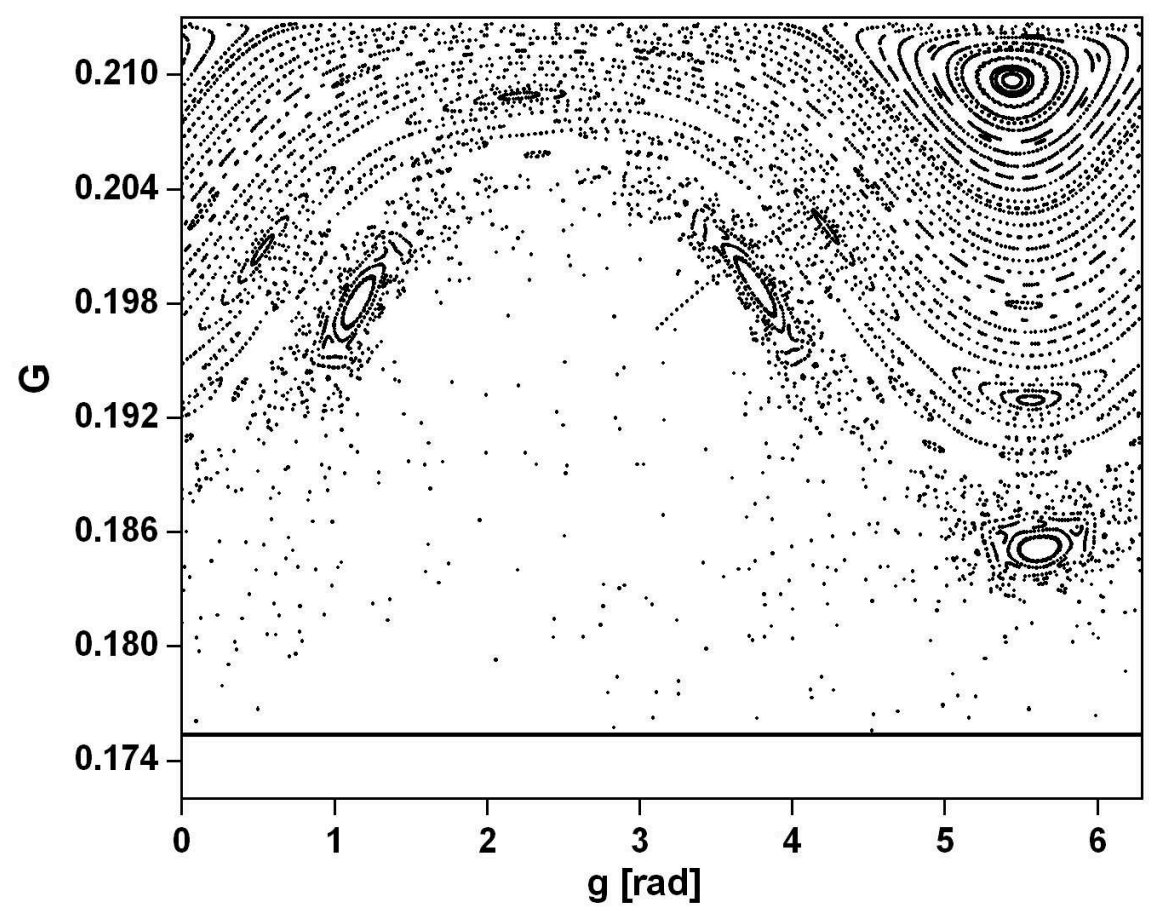

b. $\alpha=4000 \mathrm{~km}, 25^{\circ}<\mathrm{I}<43^{\circ}$

Fig. 3 Poincare sections of the ' $3 \mathrm{G}$ ' model. The horizontal, solid lines indicate the border under which the satellite collides with the surface of the Moon 

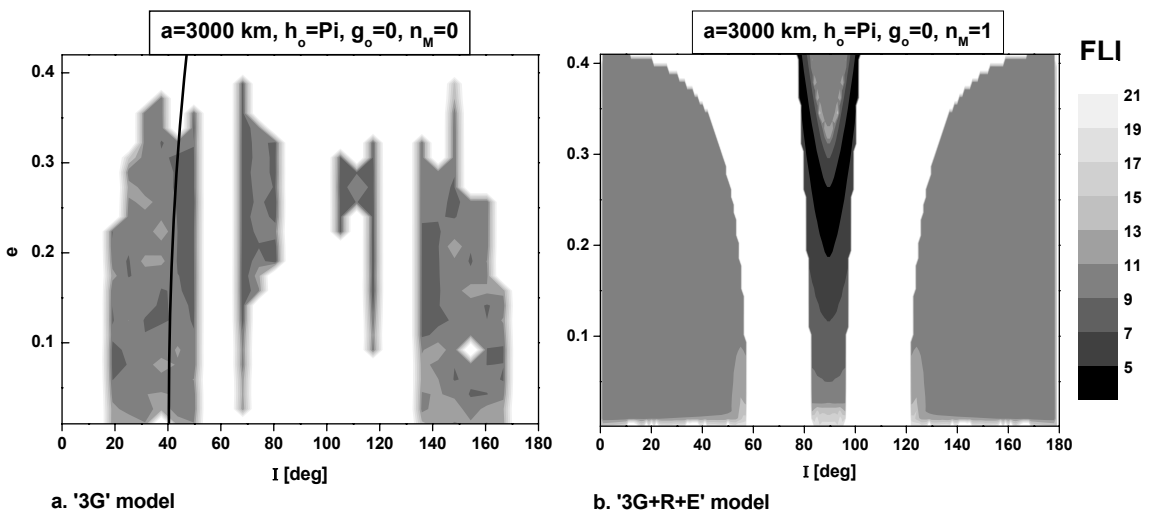

Fig. 4 FLI maps. The bold, solid line in diagram (a) represents the initial conditions of the orbits in Fig. 3a
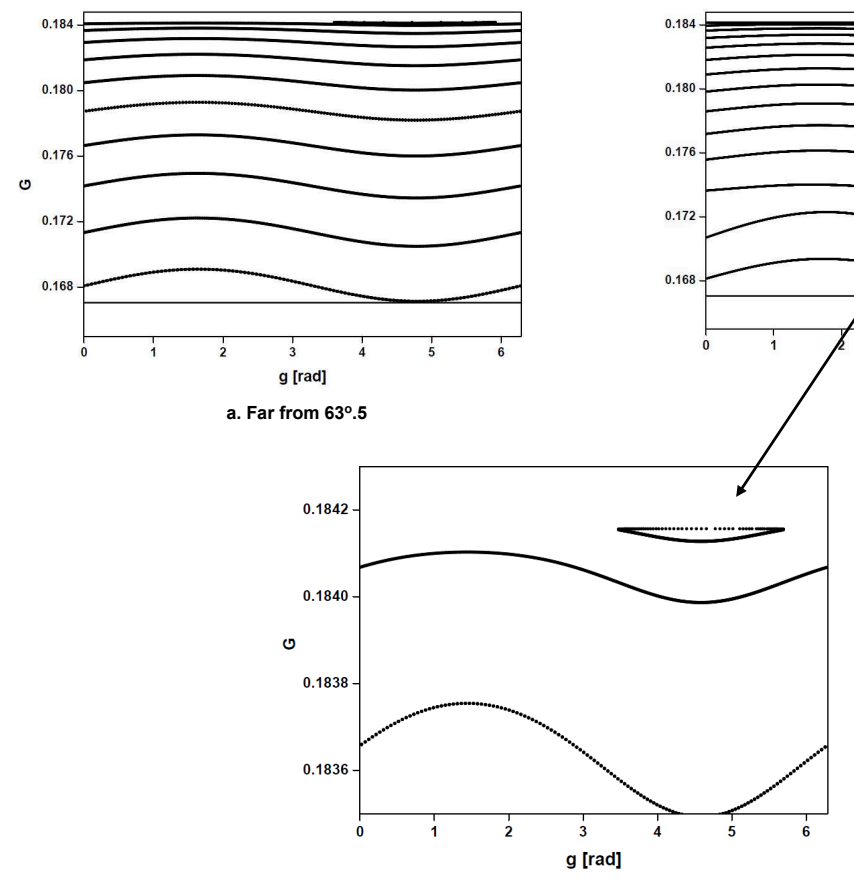

c. Zoom in the region of large $G$

Fig. 5 Poincaré sections of the ' $3 \mathrm{G}+\mathrm{R}$ ' model for $a=3000 \mathrm{~km}$, far from (a) and close to $I=63^{\circ} .5$ (b). Diagram (c) zooms in the region of large values of $G$ (small values of $e$ ), where the elliptic periodic orbits appear 

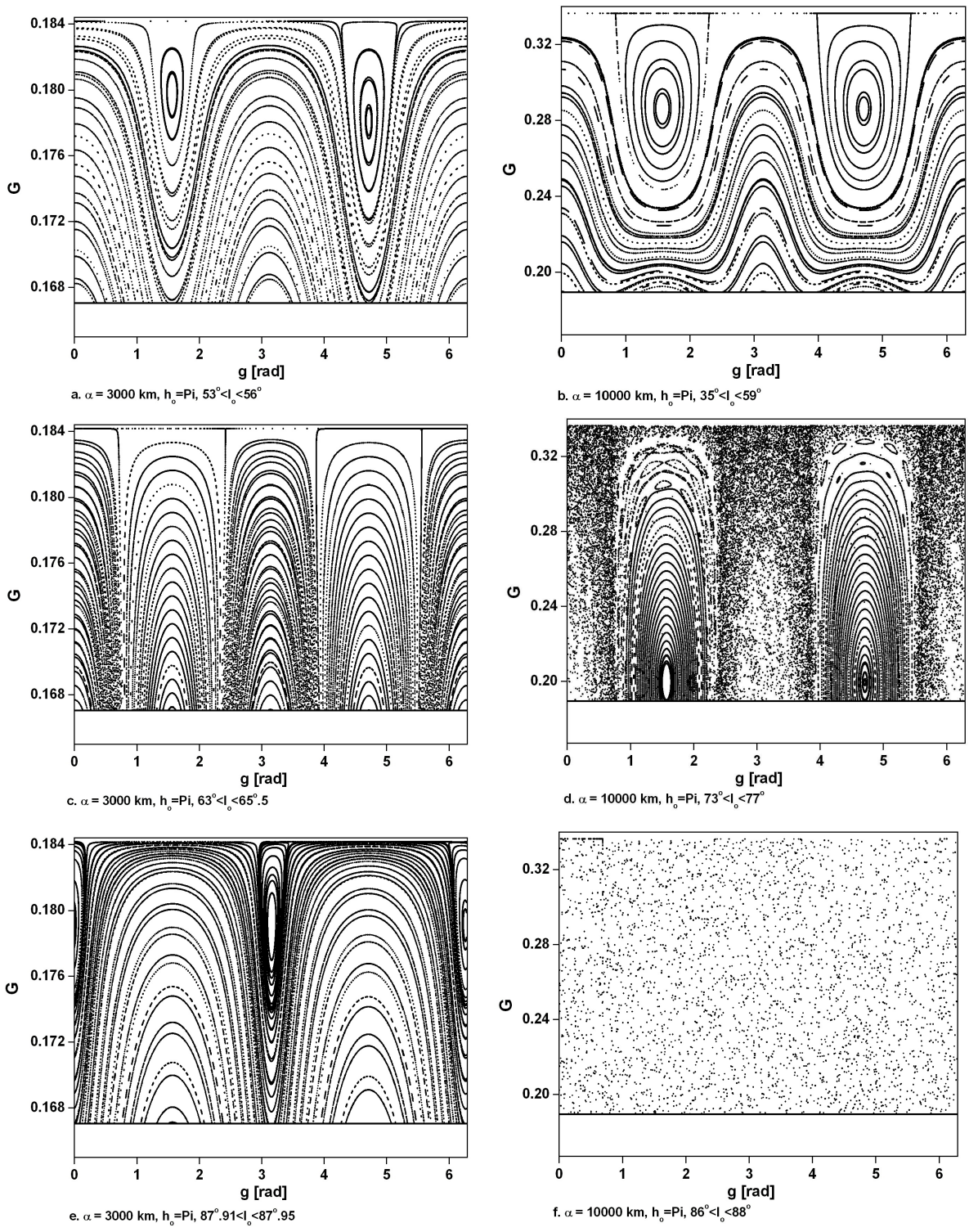

Fig. 6 Poincaré sections of the ' $3 \mathrm{G}+\mathrm{R}+\mathrm{E}$ ' model for $a=3000 \mathrm{~km}$ (left) and $a=10000 \mathrm{~km}$ (right) for three different inclination ranges

\section{Families of stable POs}

The distribution of POs in phase space is essential for understanding the dynamics of a nearly integrable system, as they form the 'backbone' of phase space. From a practical point of view, POs are essential for efficient design of lunar orbiter missions, since, as a rule, minimal spacecraft control is required in the vicinity of that kind of orbits (see Knežević and Milani 1998). 
In order to determine the initial conditions of the POs, we use differential corrections (Deprit and Henrard 1967). For a PO, we have that $g(t, T)=g(t), G(t+T)=G(t)$ and $h(t+T)=h(t)$, where $t$ is the time, $T$ is the period of the orbit and $g, h \in[0,2 \pi]$. Starting from initial conditions $\left(g_{0}, G_{0}\right), h=h_{\text {Section }}, H=f\left(g_{0}, G_{0}, h_{0}, \mathcal{H}\right)$, we find the next section point in time $t^{*}$ with coordinates $\left(g\left(t^{*}\right), G\left(t^{*}\right)\right)$. This method is based on the idea of consecutive corrections of the initial conditions, in order to ensure that at time $t^{*}$ the orbit returns at the same point on the section. This process is repeated, until succeeding the setting accuracy. In our calculations tolerance was set equal to $10^{-12}$. The method converges if we start close enough to the PO (i.e. in the region of an island).

We calculated the main, stable POs for a variety of semi-major axis values, with emphasis on the ' $3 \mathrm{G}+\mathrm{R}$ ' and ' $3 \mathrm{G}+\mathrm{R}+\mathrm{E}$ ' models, which are more realistic. The description and the diagrams that follow, refer to $a=3000 \mathrm{~km}$ and $h_{0}=\pi$.

' $3 G$ ' model: Stable POs appear in regions of regular motion and are not symmetrically distributed with respect to $I=90^{\circ}$ (see Fig. 7). The eccentricity of these POs is quite large, almost for all inclinations. Since the model has 2-degrees of freedom, the quantities $e, g$ and $I$ of POs are not expected to be constant. Instead they perform librations with amplitudes $\Delta e, \Delta g$ and $\Delta I$ (defined here as the difference between the largest and the smallest value) $0.08<\Delta e<0.28$ and $5^{\circ}<\Delta I<35^{\circ}$, while $g$ of the POs is circulating for this model almost for all inclinations. The libration amplitudes decrease as the semi-major axis of the orbit increases. The distribution of POs is 'broken' close to $I=60^{\circ}, 90^{\circ}$ and $125^{\circ}$, where the ratio $f_{g} / f_{h}\left(f_{g}\right.$ and $f_{h}$ represent the frequencies of the angles $g$ and $h$, respectively) changes resonance.

' $3 G+R$ ' model: The eccentricity of the POs remains close to zero for all inclinations, except for $I \approx 63^{\circ} .5$ and $116^{\circ} .5$, where two branches of POs emanate from the main families of the axisymmetric model (Fig. 7). By varying the energy, one of them moves to lower eccentricities and the other to higher ones, both keeping $I$ almost constant. The nearly-circular POs correspond to the island appearing at large $G$ (small $e$ ) in the Poincaré sections (see Fig. 5). The high eccentricity POs correspond to the island that appears at $I \approx 63^{\circ} .5$, i.e. correspond to the 'critical' inclination orbits of the $J_{2}$ problem. The effect of the rotation is to reduce the libration amplitudes, so that $\Delta e<0.003, \Delta I<0^{\circ} .4$. Also, $\Delta g$ is smaller than $20^{\circ}$ almost for all inclinations (libration), but it increases as $I$ tends to $0^{\circ}$ or $180^{\circ}$, where $g$ starts to perform rotations (see Fig. 8). We studied the dependence of the POs on $a$ for $R+100 \mathrm{~km} \leq a \leq 4000$ $\mathrm{km}$ with $\Delta a=100 \mathrm{~km}$, or even smaller in some intervals. Some of these results are presented on an $e-I$ diagram (Fig. 11a). The results for all the cases studied are not included for better readability of the diagram.

' $3 G+R+E$ ' model: In this case, the most complete model in our study, there appear two 'gaps' with no POs (see Fig. 7 and 4b). This is because near these gaps the eccentricity of a PO exceeds the collision value, $e_{c}$ (see Fig. 6d) and thus we do not mark it in the diagram. In the other regions, the eccentricity remains very close to zero $(e<0.001)$. As far as libration amplitudes are concerned, we find $\Delta g<10^{\circ}$, $\Delta e<0.01$ and $\Delta I<0^{\circ} .5$ (Fig. 8), slightly increasing for larger values of $a$. It is worth mentioning that for $a=R+100 \mathrm{~km}, \Delta e$ is such that the minimum distance 


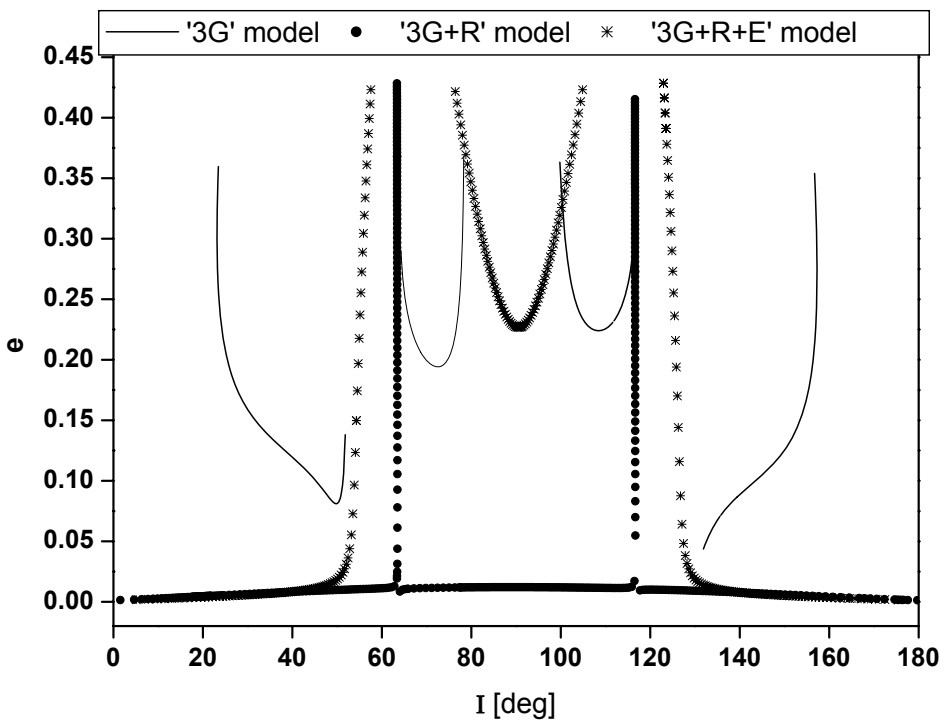

Fig. $7 e-I$ diagram of the main, stable POs for the three models studied ( $a=3000 \mathrm{~km})$

between the satellite and the surface of the Moon for polar orbits equals to $50 \mathrm{~km}$, $\left(I \approx 90^{\circ}\right)$. Again, we find the POs for different values of $a$, but now we include the cases $a=5000,10000,15000,20000 \mathrm{~km}$. Some of these results are presented in Fig. 11b, while others are not included for better readability of the diagram. Nevertheless, cases for $a>5000 \mathrm{~km}$ have similar behavior as those for $a=5000 \mathrm{~km}$. For small values of $a$ (for example $a=R+500 \mathrm{~km}$ ), the 'gap' of POs around $I \approx 63^{\circ} .5$ is narrow and it widens as $a$ increases, an effect attributed to perturbations by Earth. Moreover, for $a \leq 2900 \mathrm{~km}$ we find polar POs at small eccentricities, while for larger semi-major axes the eccentricity of the POs around $I=90^{\circ}$ has higher values. The time evolution of some POs for $a=3,000 \mathrm{~km}$ and $a=R+100 \mathrm{~km}$ is presented in Figs. 9. and 10, respectively (the orbits on the right are under the additional effect of the Earth, while these on the left are not). It is quite clear that for $a$ values around $3,000 \mathrm{~km}$ the effect of Earth modifies the shape of the orbits, something that is not true for low semi-major axis values. In all cases, the libration amplitude of $e$ is smaller than 0.02 .

As mentioned earlier in this paper, Abad et al. (2009) have done similar work, for different semi-major axis values, taking into account coefficients $J_{2}$ and $J_{7}$. The problem they studied is of 1-degree of freedom, so they searched for frozen orbits (i.e. $e$ and $g$ remain constant with time). In our case there is an additional degree of freedom because of the tesseral and sectorial coefficients, so we study POs instead of frozen orbits, the orbital elements of which perform oscillations. 

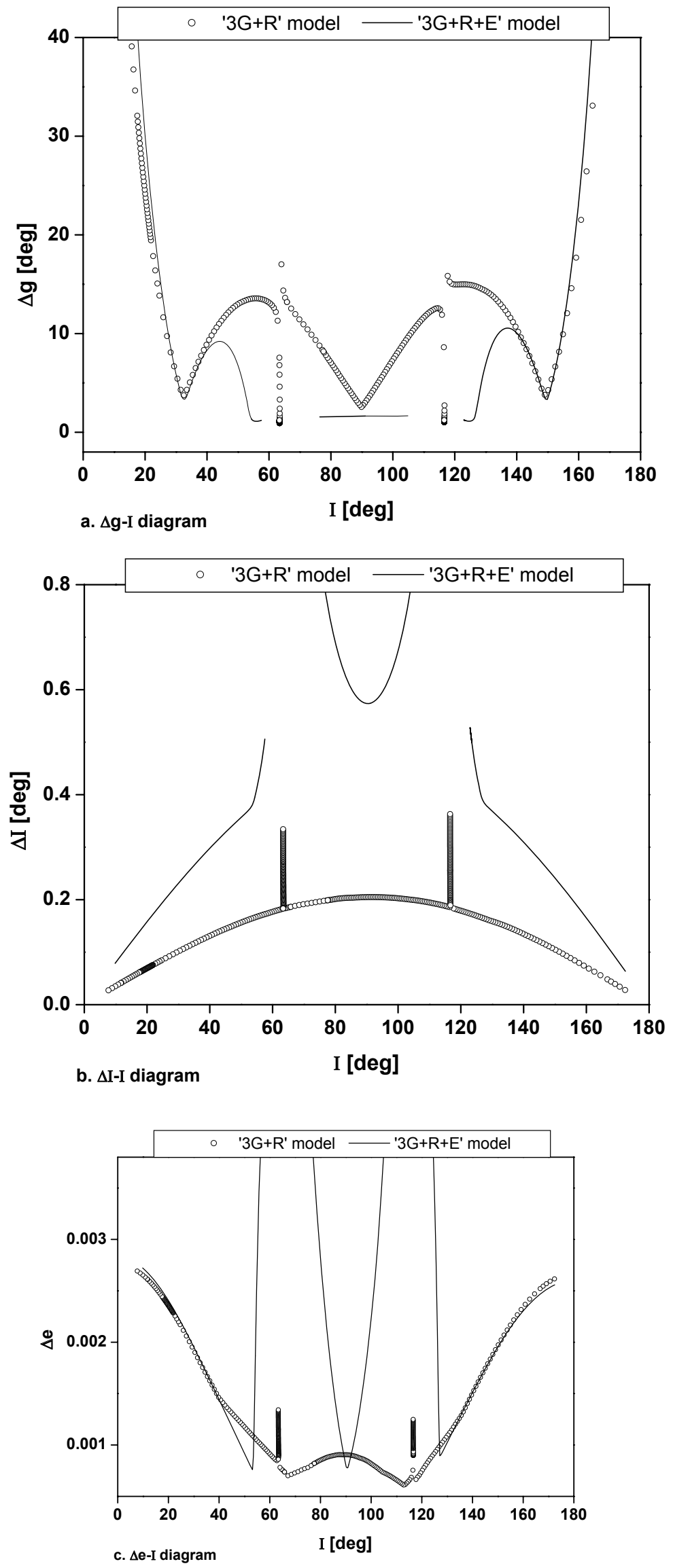

Fig. $8 \Delta g, \Delta I, \Delta e-I$ diagrams of the main families of $\mathrm{POs}$ for the ' $3 \mathrm{G}+\mathrm{R}$ ' and ' $3 \mathrm{G}+\mathrm{R}+\mathrm{E}$ ' models for $a=3000 \mathrm{~km}$ 


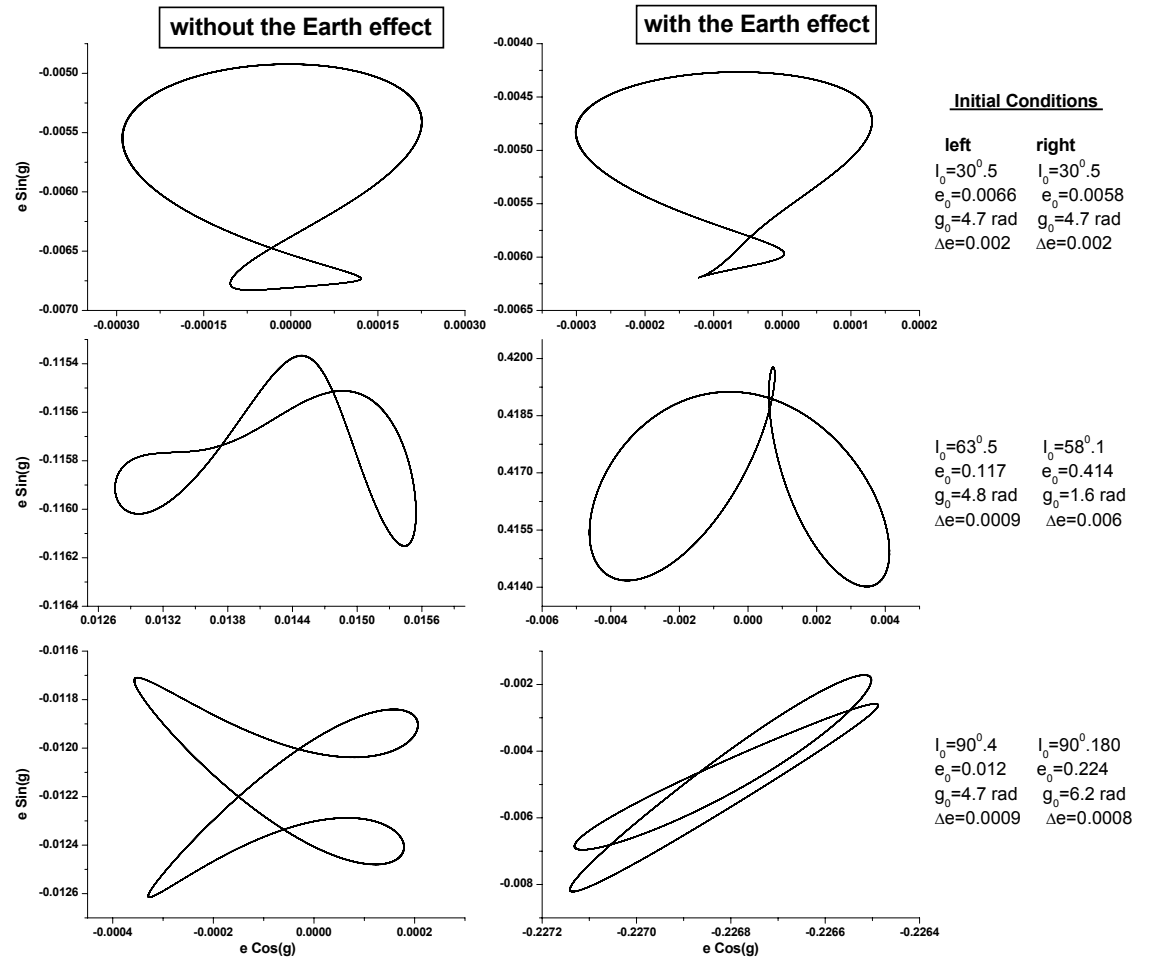

Fig. 9 Time evolution of some POs, for $a=3000 \mathrm{~km}$ and $h_{0}=\pi$, for the ' $3 \mathrm{G}+\mathrm{R}$ ' (left) and the ' $3 \mathrm{G}+\mathrm{R}+\mathrm{E}$ ' models.

\section{Conclusions}

We have shown that when $3^{r d}$-degree terms are included in the lunar potential, the secular behavior of the orbits changes significantly, as compared with the ' $J_{2}+C_{22}\left(+n_{M}\right)$ ' models, for which the motion, as a rule is regular. When the rotation of the Moon is not taken into account, the phase space contains extensive chaotic or collision regions. The inclusion of the lunar rotation weakens these phenomena, so that the motion appears to be mostly regular. When the effect of Earth is added, chaotic or collision regions appear again in phase space. There is an obviously different behavior between $a<3000$ $\mathrm{km}$, where the $3^{r d}$-degree lunar gravity terms are dominant, and $a>3000 \mathrm{~km}$, where the effect of Earth is more important. Note that we have selected the value $3000 \mathrm{~km}$ after extensive numerical computations, as an easy to remember approximate value. The behavior of the orbits changes close to that semi-major axis value and not exactly at that value.

The main families of POs emanate from the families of POs of the axisymmetric problem $\left({ }^{\prime} J_{2}+J_{3}\right.$ '). These are the equivalent of nearly-circular 'frozen-eccentricity' orbits $(\forall I)$ and 'critical inclination' orbits $\left(I \approx 63^{\circ} .5\right.$ and $\left.116^{\circ} .5\right)$. In the '3G' model, POs usually appear at large eccentricities, while in the ' $3 \mathrm{G}+\mathrm{R}$ ' model there exist elliptic POs for every $I$. For $I$ close to $63^{\circ} .5$ new POs appear for higher eccentricities. 

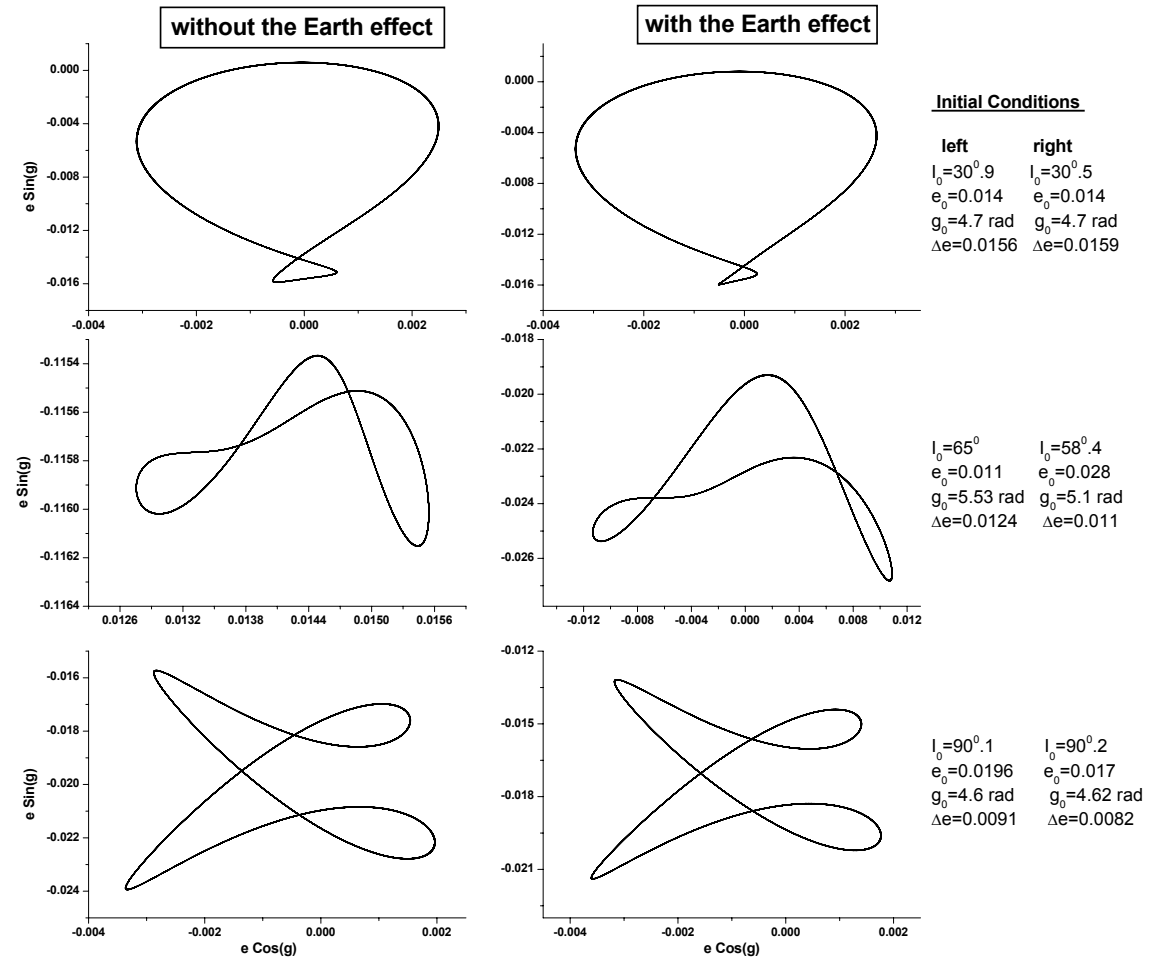

Fig. 10 Time evolution of some POs, for $a=R+100 \mathrm{~km}$ and $h_{0}=\pi$, for the ' $3 \mathrm{G}+\mathrm{R}$ ' (left) and the ' $3 \mathrm{G}+\mathrm{R}+\mathrm{E}$ ' models.

When Earth is included, there are (main, stable) POs for every inclination, except for a gap of chaotic/collision orbits. $G$ and $I$ are no longer integrals of motion, but their variations $(\Delta I, \Delta e, \Delta g)$ are small, when $n_{M} \neq 0$. These POs can be used as the basis for an efficient design of artificial satellite orbits, requiring minimal control, as already discussed in Knežević and Milani (1998).

In the future, we intend to extend this work to the numerical computation of POs in the full gravitational potential of the Moon. To this end, it would be desirable to have a closed form relation (in $e$ and $I$ ) for the $1^{\text {st }}$-order averaged Hamiltonian (De Saedeleer has done this for the zonal problem, 2005). As the computation of the POs will become quite cumbersome, it would be desirable to have an analytic (perturbative) method that would be able to provide an accurate determination of the POs location. This work is under way and we hope to report on these issues in a forthcoming paper.

\section{References}

1. Abad, A., Elipe, A., Tresaco, E., Analytical model to find frozen orbits for a lunar orbiter, JGCD, 32(3), 888-898 (2009)

2. Bertotti, B., Farinella, P., Vokrouhlicky, Physics of the Solar System, 42-59. Kluwer Academic Publishers, Dordrecht/Boston/London (2003) 

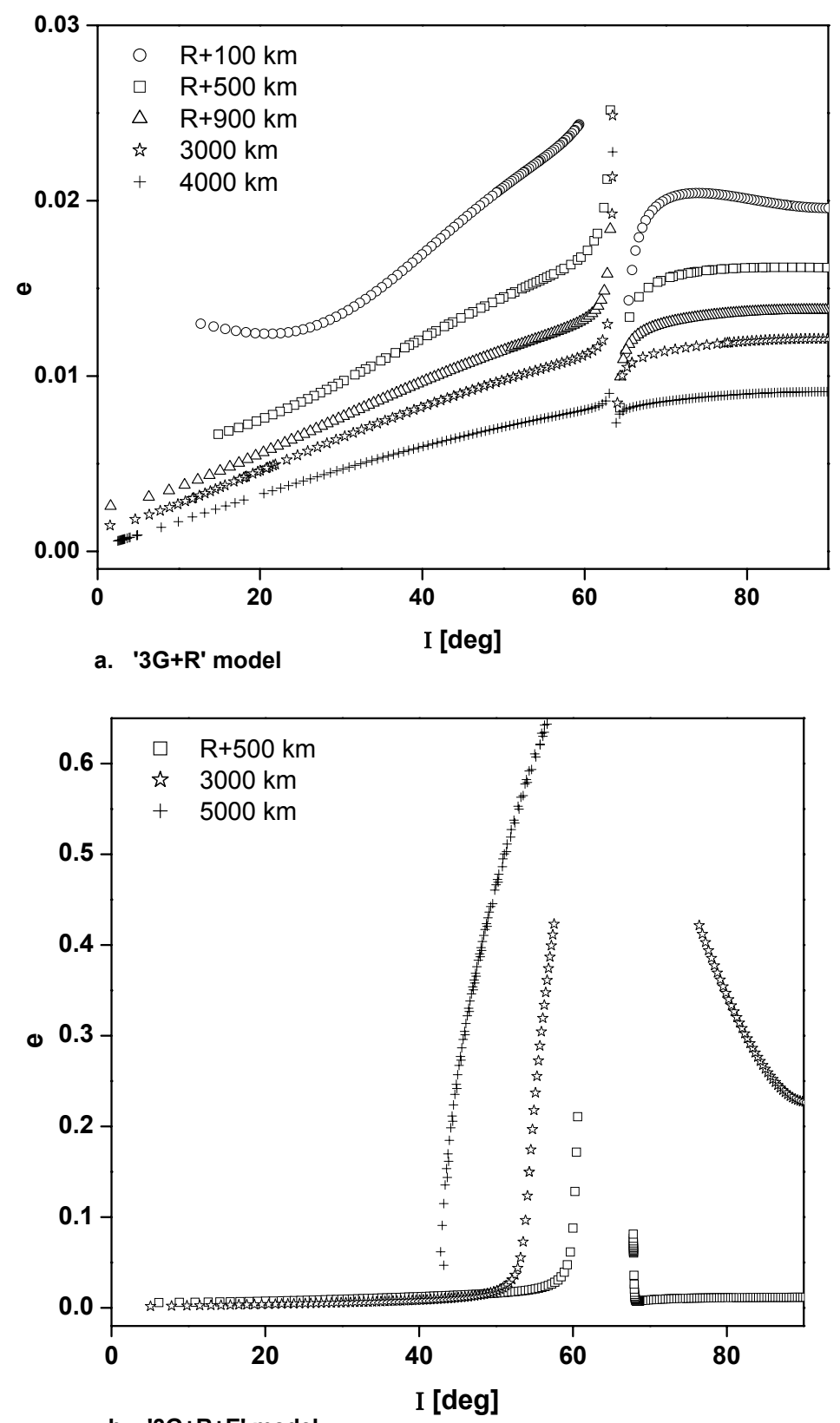

Fig. $11 e-I$ diagrams of the main POs without (a) and with (b) the effect of the Earth for various semi-major axis values 
3. Coffey, S.L., Deprit, A., Deprit, E., Frozen Orbits close to an Earth-like planet, Celest. Mech. Dyn. Astr., 59, 37-72 (1994)

4. Coffey, S.L., Deprit, A., Miller, B.,R., The critical inclination in artificial satellite theory, 39, 365-406 (1986)

5. Cutting, E., Born, G.H., Frautnick, J.C., Orbit Analysis for SEASAT-A, Journal of the Astronautical Sciences, 26(4), 315-342 (1978)

6. Deprit, A., Henrard, J., Natural Families of Periodic Orbits, AJ, 72, 158-172 (1967)

7. De Saedeleer, B., Complete Zonal problem of the artificial satellite: Generic compact analytic first order in closed form, Celest. Mech. Dyn. Astr., 91, 239-268 (2005)

8. De Saedeleer, B., Théorie analytique fermée d'un satellite artificiel lunaire pour l'analyse de mission, Presses Universitaires de Namur, Belgique (2006a)

9. De Saedeleer, B., Analytical theory of lunar artificial satellite with third body perturbations, Celest. Mech. Dyn. Astr., 95, 407-423 (2006b)

10. De Saedeleer, B., Henrard, J., The combined effect of $J_{2}$ and $C_{22}$ on the critical inclination of a lunar orbiter, Advances in Space Research, 37, 80-87 (2006)

11. Elipe, A., Lara, M., Frozen orbits in the tesseral artificial satellite theory, Proceedings of the $12^{t h}$ International Symposium on 'Space Flight Dynamics', ESA Special Publication, 403, 397-402 (1997)

12. Froeschlé, C., Gronczi, R., Lega, E., The fast Lyapunov indicator: a simple tool to detect weak chaos. Application to the structure of the main asteroidal belt, Planetary and Space Science, 45, 881-886 (1997)

13. Garfinkel, B., The global solution in the problem of the critical inclination, Celest. Mech., 8, (1973)

14. Hughes, S., The 'Critical Inclination': Another Look, Celest. Mech., 25, 235-266 (1981)

15. Jupp, A.H., The Critical Inclination problem - 30 years of progress, Celest. Mech., 43, $127-138(1988)$

16. Knežević, Z., Milani, A., Orbit maintenance of a lunar polar orbiter, Planet space Sci., 46, 1605-1611 (1998)

17. Lara, M., Deprit, A., Elipe, A., Numerical continuation of families of frozen orbits in the zonal problem of artificial satellite theory, Celest. Mech. Dyn. Astr., 62, 167-181 (1995)

18. Lara, M., De Saedeleer, B., Ferrer, S., Preliminary design of low lunar orbits, Proceedings of the $21^{\text {st }}$ International Symposium on Space Flight Dynamics, edited by CNES, Sep-Oct 2009, (2009)

19. Lega, E., Froeschlé, C., On the relationship between fast lyapunov indicator and periodic orbits for symplectic mappings, Celest. Mech. Dyn. Astr., 81, 129-147 (2001)

20. Liu, L., Innanen, K.A., Problems of critical inclination and commensurability in the motion of artificial satellites, Chin. Astron. Astrophys., 10, 245-251 (1986)

21. Murray, C., Dermott, S., Solar System Dynamics. Cambridge University Press, United Kingdom (1999)

22. Roy, A.E., Orbital Motion, 283-286. Adam Hilger LTD, Bristol (1982)

23. Sidi, M.J., Spacecraft Dynamics and Control, 34-35. Cambridge University Press, USA (2002)

24. Tzirti, S., Tsiganis, K., Varvoglis, H., Quasi-critical orbits for artificial lunar satellites, Celest. Mech. Dyn. Astr., 104, 227-239 (2009)

25. Vallado, D.A., Fundamentals of Astrodynamics and Applications. Kluwer Academic Publishers, Dordrecht/Boston/London (2001) 